\title{
"As soon as the bat met the ball, I knew it was gone": Outcome prediction, hindsight bias, and the representation and control of action in expert and novice baseball players
}

\author{
RoB GraY \\ Arizona State University, Mesa, Arizona \\ SIAN L. BEILOCK \\ University of Chicago, Chicago, Illinois \\ AND \\ ThOMAS H. CARR \\ Vanderbilt University, Nashville, Tennessee
}

\begin{abstract}
A virtual-reality batting task compared novice and expert baseball players' ability to predict the outcomes of their swings as well as the susceptibility of these outcome predictions to hindsight bias-a measure of strength and resistance to distortion of memory for predicted action outcomes. During each swing the simulation stopped when the bat met the ball. Batters marked where on the field they thought the ball would land. Correct feedback was then displayed, after which batters attempted to remark the location they had indicated prior to feedback. Expert batters were more accurate than less-skilled individuals in the initial marking and showed less hindsight bias in the postfeedback marking. Furthermore, experts' number of hits in the previous block of trials was positively correlated with prediction accuracy and negatively correlated with hindsight bias. The reverse was true for novices. Thus the ability to predict the outcome of one's performance before such information is available in the environment is not only based on one's overall skill level, but how one is performing at a given moment.
\end{abstract}

Predicting an action's outcome before complete sensory feedback has been received is highly advantageous in sensorimotor skills. A basketball player is more likely to rebound a miss if that miss is predicted before the ball hits the rim. And, in baseball, knowing where one's hit will land gives the batter a base-running advantage.

Although one might suppose that high-level athletes are better at such predictions than novices, this has neither been directly tested nor has a mechanism underlying an expertise advantage (if it does exist) been established. Insight into such skill level differences will not only illuminate the mechanisms governing outcome prediction, but may provide new insight into the cognitive processes supporting execution across the learning continuum.

How might outcomes be predicted? By definition, direct sensory feedback from the environment is not available. Therefore, outcome cues must be extracted from the information that $i$ available when the prediction is made: Internally generated information about the performance itself and/or expectations about anticipated performance outcomes.
Using the former information source, performers may engage in "execution monitoring" (attending to the step-bystep unfolding of motor execution; Beilock \& Carr, 2001) and make judgments about how successfully those steps are being taken. Another possibility is that performers engage in "expectation monitoring"-attending to expectations about what sensory feedback should be coming from the environment if the intended action unfolds as planned.

"Expectation monitoring" may seem like magical thinking - expecting an outcome doesn't mean it will happen. However, Prinz's (1997) “common coding” theory provides a principled underpinning for just such an explanation of how predictions might be made. Common coding suggests that actions are planned and controlled by their intended effects. As in earlier feedback-based control theories (see Powers, 1978), Prinz proposes that the governing nodes in motor control hierarchies are intended environmental changes that actions should create. Therefore, directing attention toward effects of one's movements is more beneficial for performance than focusing on the movements themselves-because the effects allow 
the most successful movements to be generated in the first place. Note, however, this notion assumes stored links between actions and their associated effects (Prinz, 2003). Thus, although intended outcomes may be tightly coupled with movements that instantiate these effects among experienced performers, this may not necessarily hold for novices.

Attending to effects is characterized by Wulf and Prinz (2001) as external attentional focus whereas "execution monitoring" is characterized as internal attentional focus. And, in tasks ranging from golf putting to baseball batting, it has been demonstrated that skilled performers benefit from conditions that limit execution monitoring (e.g., simultaneously performing an unrelated secondary task or operating under "speed" instructions to perform rapidly). This contrasts with conditions that prompt execution monitoring (e.g., reporting on a specific mechanical aspect of execution), which hurts skilled performers (Beilock, Bertenthal, McCoy, \& Carr, 2004; Beilock, Carr, McMahon, \& Starkes, 2002; Gray, 2004; Jackson, Ashford, \& Norsworthy, 2006).

These findings indicate that execution monitoring is bad for experts, but they do not say what experts should be monitoring instead. Common-coding theory makes a prediction. Experts should be attending to information about anticipated perceptual feedback generated by the performance. And, such information should prove quite useful in predicting what actually occurs-especially to the extent that one has established a strong link between action outcomes and the movements that produce such effects.

In contrast, several studies have demonstrated that novice performance in sports such as soccer, golf, and field hockey deteriorates when attention is paid to anything other than the mechanics of execution (Beilock, Wierenga, \& Carr, 2002; Gray, 2004; Jackson et al., 2006; PerkinsCeccato, Passmore, \& Lee, 2003). One might imagine, then, that novices attend to different kinds of information in real-time than experts, and if novices can make outcome predictions at all, their predictions are based on different kinds of information than experts' predictions.

Moreover, recent work suggests that performers' attention not only varies with overall skill level, but also with current performance level. Gray (2004) showed that expert batters allocate more attention to the mechanics of execution during periods of poorer performance ("slumps") and more attention to the external environment during periods of better performance ("streaks"). The opposite is true for novices. If a slump for skilled performers represents increased allocation of attention to the steps of performance rather than its outcome, then experts should be worse at predicting the results of their actions when performing poorly. In contrast, if a slump for novices represents increased attention to outcome-relevant cues, then novices should show improved outcome prediction when performing poorly. Thus, to the extent that experts attend more to distal outcome-relevant information during the process of generating their performance than do novices (who must focus on the proximal component steps of execution), experts should be better able to predict their performance outcomes prior to feedback. This should be especially true during successful performance when attention is likely directed even more to the anticipated outcome of execution rather than execution itself.

To investigate these ideas, novice and expert baseball players performed a simulated hitting task and made predictions about where the ball would land before receiving feedback about where it actually landed. To assess such predictions' robustness, a hindsight bias paradigm was used to measure memory strength. Hindsight bias (Fischhoff, 1975) is the tendency for individuals to reconstruct knowledge of "what seemed likely to happen" (foresight) after receiving information about "what did happen" (hindsight). To the extent that one is attending to outcome-relevant information, and such attention increases the strength of one's memory for outcome predictions (Craik, Govoni, Naveh-Benjamin, \& Anderson, 1996), memory for the prediction should be less susceptible to distortion by feedback information.

\section{METHOD}

\section{Participants}

Sixteen males (19-26 years) participated. Experts $(n=8)$ were collegiate baseball players (competitive experience, $M=14.2, S E=$ 0.5 years). Novices $(n=8)$ played less and never at the college level ( $M=5.1, S E=0.6$ years). Novices without competitive experience were not used because they could not reliably hit the simulated ball. In our investigation, 'expertise' combines differences in experience per se with differences in aptitudes and motivations. While this is ecologically valid for examinations of real-world expertise, future research could separate these factors as possible causal variables.

\section{Apparatus}

A modified version of Gray's (2002a, 2002b, 2004) batting simulation was used. Participants swung a real bat at a simulated approaching baseball. The image of the ball, pitcher and playing field (Figure 1) were projected on a $2.11 \mathrm{~m}(\mathrm{~h}) \times 1.47 \mathrm{~m}$ (v) screen using a Proxima $6850+$ LCD projector. Batters stood on their preferred batting side of a standard $0.45 \times 0.45 \mathrm{~m}$ home plate on a greencarpeted floor $2.5 \mathrm{~m}$ in front of the screen. Mounted on the bat's end was a sensor from a Fastrak (Polhemus) position tracker. The $x, y, z$ position of the bat's end was recorded at $120 \mathrm{~Hz}$.

Motion toward the batter was simulated by increasing the ball's angular size. Drop due to gravity (ignoring air resistance and spin) was simulated by varying the ball's height. All pitches were strikes crossing the center of the plate. Pitch speed varied randomly from trial to trial from 30 to $40 \mathrm{~m} / \mathrm{sec}(67-90 \mathrm{mph})$. Although this is a simplified version of real batting, where there can also be variation in the ball's lateral position, judgment of height is more difficult and causes more performance errors (Bahill \& Karnavas, 1993). Therefore, this simplified task incorporated the most demanding perceptual batting judgment.

Each trial began with a 10 -sec view of the playing field and the pitcher in the starting pose (Figure 1, top panel). The pitcher executed a delivery lasting roughly $3 \mathrm{sec}$ before releasing the ball. The ball's position in the simulation was compared with the bat's recorded position in real-time to detect bat/ball collisions. Estimated static positional tracking precision $(<0.2 \mathrm{~mm})$ was derived from the standard deviation of 50 samples with the sensor at a constant position. System dynamic precision $(<1 \mathrm{~mm})$ was estimated using the method described by (Tresilian \& Lonergan, 2002).

\section{Procedure}

Participants began with three 20-trial practice blocks with full feedback and no outcome marking, then three 20 -trial blocks prac- 

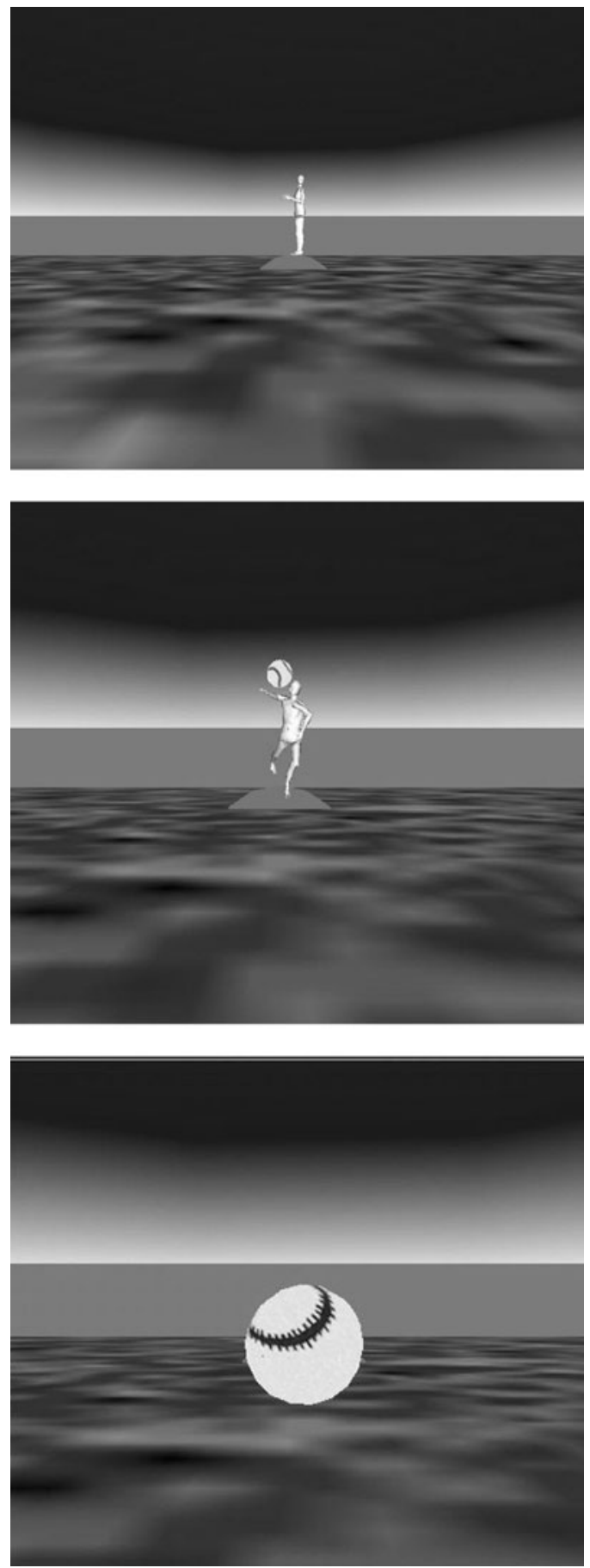

Figure 1. Visual display in the batting simulation.

ticing marking the outcome of each swing. Participants then completed 10 blocks of 20 experimental trials with outcome marking and delayed feedback (see below) with a 5-min interblock interval.

Outcome marking occurred as follows: A 3-sec blank screen appeared when the virtual ball and bat were the same distance from the screen (i.e., the point of contact or, for misses, the point at which ball crossed over/under bat). The batter then saw the empty field, with a yellow cone at the bottom center. Using a mouse placed on a table behind home plate, batters moved the cone to the location they felt the ball would land, clicking the left mouse button when satisfied, keeping their eyes on the screen at all times. For home runs batters marked where the ball would cross the outfield fence. For foul balls they marked where the ball would leave the playing field. For ground balls, batters marked where the ball would come to a stop. For a miss, they left the mouse at bottom-center of the display. When the mouse button was pressed, the $x-y$ location of the marker was recorded and the screen was blanked for $3 \mathrm{sec}$. Correct visual and auditory feedback for the swing was then displayed.

For no contact between bat and ball, audio of an umpire saying "strike" was played. If contact was detected the "crack" of a bat was played and the location of the bat, bat speed, ball speed and bat angle were used to visually simulate the ball flying off the bat into the playing field. For foul balls audio of an umpire saying "foul ball" was played. For home runs (fair balls that traveled further than $107 \mathrm{~m}(350 \mathrm{ft})$, audio of an announcer's home run call was played. For balls beyond the infield $(130 \mathrm{ft})$ but not home runs, audio of a crowd cheering was played. Finally, for balls that did not travel out of the infield audio of a crowd groaning was played. This auditory feedback matched the definition of successful hits (see Data Analysis).

Following feedback, the display blanked for $20 \mathrm{sec}$ then the field and marker cone reappeared. Batters were instructed to remark the same position they had done previously. They were further instructed that the study was testing their memory and they should try not to let the feedback influence their second marking.

\section{Control Conditions}

To determine the magnitude of hindsight bias we compared data from the procedure described above with data from two control conditions:

Memory decay condition. The extent to which memory for predictions about swing outcome decayed between markings. This condition was identical to above except that a blank screen (rather than outcome feedback) appeared between the two markings.

Match-the-feedback condition. The extent to which participants could mark the landing position of the ball they had seen in the feedback condition. The procedure was identical to outcome marking except that during the second marking interval participants were instructed to mark the position they saw the ball land during the feedback, rather than the position they predicted the ball would land before the feedback.

The two control conditions were performed after the experimental trials, with order counterbalanced across participants.

\section{Data Analysis}

First and second marking positions were compared to actual outcomes (computed by the simulation) to calculate marking error. Using the location of the marker $\left(x_{m}, y_{m}\right)$ and the calculated location of the simulated ball $\left(x_{a}, y_{a}\right)$, we calculated a radial distance error (d) using the formula

$$
d=\sqrt{\left(x_{m}-x_{a}\right)^{2}+\left(y_{m}-y_{a}\right)^{2}} .
$$

Pilot testing revealed the range in $x_{a}$ and $y_{a}$ was significantly smaller for experts than for novices (i.e., novices spread the ball around more than experts, largely due to more foul balls and misses). This creates a potential problem when comparing outcome marking as any difference in accuracy could be due to the smaller range of possible predicted locations among experts. Therefore, we also scaled radial distance error by the pooled variance in swing outcomes. Scaled marking error (SME) was computed as follows:

$$
S M E \equiv \frac{d_{\text {mean }}}{\sqrt{\left[\sum\left(x_{a}-\bar{x}_{a}\right)^{2} /(t-1)\right]+\left[\sum\left(y_{a}-\bar{y}_{a}\right)^{2} /(t-1)\right] / 2}},
$$

where $d_{\text {mean }}$ is mean radial error and $t$ is number of trials. To quantify batting performance we divided outcomes into four categories: (1) HITS, defined as fair balls (including ground balls) traveling 
Table 1

Proportions of Actual and Predicted Swing Outcomes

\begin{tabular}{lccccc}
\hline & \multicolumn{2}{c}{ Novices } & & \multicolumn{2}{c}{ Experts } \\
\cline { 2 - 3 } \cline { 5 - 6 } Outcome & Actual & Predicted & & Actual & Predicted \\
\hline Hit & .25 & .33 & & .49 & .60 \\
Infield & .18 & .09 & & .19 & .07 \\
Foul & .14 & .19 & & .17 & .22 \\
Miss & .43 & .39 & & .16 & .11
\end{tabular}

Note-Hits were higher for experts than for novices [actual, $t(14)=$ $10.3, p<.001$; predicted, $t(14)=19.2, p<.001]$, whereas misses were higher for novices than for experts [actual, $t(14)=22.5, p<.001$; predicted, $t(14)=27.8, p<.001]$.

beyond the infield, (2) INFIELDS, fair balls stopping in the infield, (3) FOULS, and (4) MISSES.

\section{RESULTS}

\section{Batting Performance}

Table 1 shows actual and predicted outcomes for novices and experts. In both, mean proportion of hits was higher for experts than novices whereas proportion of misses was higher for novices than experts.

\section{Prediction Accuracy and Hindsight Bias}

Figure 2 shows mean preoutcome-feedback and postoutcome-feedback radial marking errors. A 2 (preoutcome-feedback, postoutcome-feedback) $\times 4$ (hit, infield, foul, miss) $\times 2$ (novices, experts) ANOVA revealed a main effect of marking interval $[F(2,56)=$ $37.5, p<.001]$. Errors were lower for postoutcome markings after feedback (mean radial error; $M R E=7.1$ ) than preoutcome markings representing initial predic- tions $(M R E=11.2)$-a substantial hindsight bias effect (38\%). One might wonder whether participants tried to duplicate their original markings at all, or merely relied on memory of the feedback to make the second marking. Data from the match-the-feedback control condition (Figure 2) suggests this was not the case. MRE in the matchthe-feedback condition was 1.9 , significantly lower than the value of 7.1 observed in the postoutcome markings $[t(14)=3.4, p<.01]$. Figure 2 also shows mean radial errors for the memory decay control condition. MRE (13.7) in this condition was higher than postoutcome markings $[t(14)=4.7, p<.01]$, and slightly but not significantly higher than the original preoutcome marking error. The higher error observed when participants relied on memory for predictions alone in the absence of feedback confirms that when feedback was provided, memory for predictions shifted toward the feedback.

The main effect of expertise $[F(1,63)=29.9, p<.001]$ was also significant. Experts $(M R E=6.8)$ produced smaller errors than novices $(M R E=11.5)$. Focusing specifically on preoutcome markings shows a large expertnovice difference, demonstrating the impact of expertise on initial prediction accuracy.

Finally, there was an expertise $\times$ marking interval interaction $[F(1,56)=7.1, p<.01]$. The difference between pre- and postoutcome marking errors was smaller for experts (mean reduction $=2.4$, approximately $30.0 \%$ ) than novices (mean reduction $=5.7$, approximately $39.7 \%$ ). As shown in Figure 2, this expertise effect on hindsight bias held in all outcome categories.

Similar results were obtained for SMEs, suggesting that marking accuracy differences between experts and novices were not due to differences in the variability of

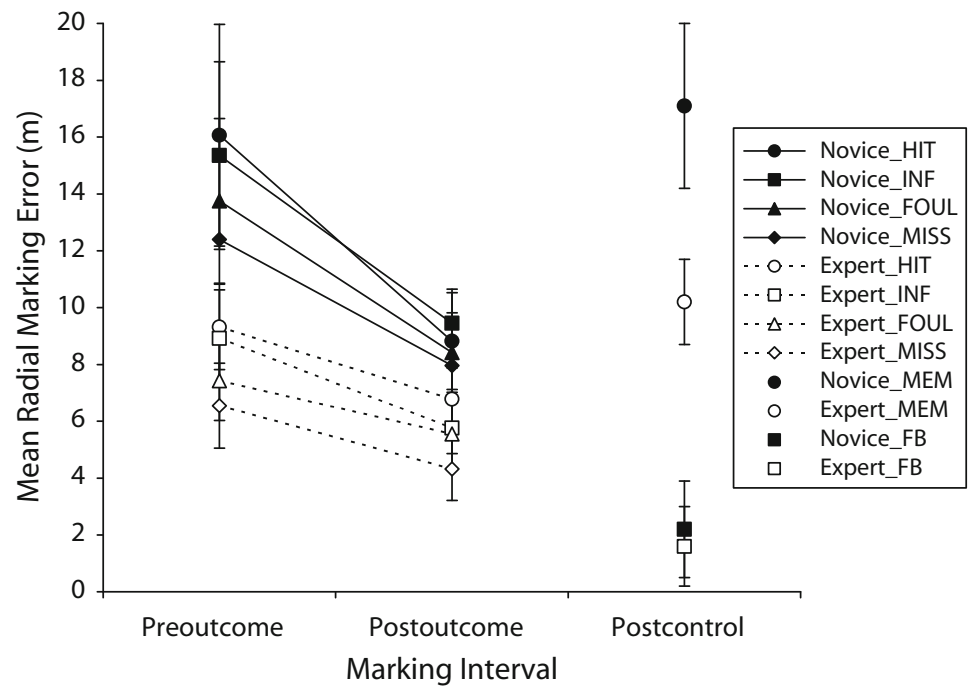

Figure 2. Mean preoutcome and postoutcome radial errors for novices and experts. Error bars are standard errors. $E \_m e m$ and $N \_m e m$ is the memory control condition in which experts and novices, respectively, remarked their original prediction without intervening feedback about the actual outcome. E_FB and N_FB is the match-the-feedback control condition in which experts and novices, respectively, received feedback and then marked the actual outcome that had just been presented in the feedback. 
Table 2

Correlations Between Preoutcome Marking Errors and Current Performance Level for Each Outcome

\begin{tabular}{llllll}
\hline & \multicolumn{2}{c}{ Novices } & & \multicolumn{2}{c}{ Experts } \\
\cline { 2 - 3 } \cline { 5 - 6 } Outcome & \multicolumn{1}{c}{$M R E$} & \multicolumn{1}{c}{$S M E$} & & \multicolumn{1}{c}{ MRE } & \multicolumn{1}{c}{$S M E$} \\
\hline Hit & $r(9)=.53$ & $r(9)=.61^{*}$ & & $r(13)=-.55^{*}$ & $r(13)=-.59^{*}$ \\
Infield & $r(10)=.43$ & $r(10)=.33$ & & $r(11)=-.50$ & $r(11)=-.42$ \\
Foul & $r(10)=.23$ & $r(10)=.21$ & & $r(10)=-.12$ & $r(10)=.03$ \\
Miss & $r(13)=-.52^{*}$ & $r(13)=-.56^{*}$ & & $r(7)=.54$ & $r(7)=.50$ \\
${ }^{*} p<.05$. & & & & &
\end{tabular}

swing outcomes. $S M E$ s were as follows: novices/pre $=$ $2.8(S D=0.35)$, novices/post $=1.6(S D=0.21)$, for a hindsight bias error reduction of $42.9 \%$; experts/pre $=2.1$ $(S D=0.18)$, and experts/post $=1.4(S D=0.17)$, for a hindsight bias error reduction of $33.3 \%$. An ANOVA performed on the $S M E$ data revealed main effects of marking interval $[F(2,56)=27.7, p<.001]$, outcome $[F(3,56)=15.6, p<$ $.001]$, expertise $[F(1,63)=24.2, p<.001]$, and an interval $\times$ expertise interaction $[F(1,56)=8.3, p<.01]$.

Separating the preoutcome radial marking error into its $x$ and $y$ components demonstrated that both experts and novices produced significantly smaller marking errors for ball direction (i.e., $x_{m}-x_{a}$ ) as compared with distance (i.e., $y_{m}-y_{a}$ ). Data were as follows. Experts: mean $x$ error $=$ 4.2 , mean $y$ error $=7.1[t(14)=5.1, p<.01]$. Novices: mean $x$ error $=8.5$, mean $y$ error $=12.2[t(14)=4.9, p<$ $.01]$. This finding is consistent with the proposal that batters are better at judging the timing of their swing relative to ball speed (which primarily determines the direction the ball travels) than the height of their swing relative to ball height (which largely determines the distance the ball travels; Bahill \& Karnavas, 1993; Gray, 2002a).

\section{Current Performance Level, Prediction Accuracy, and Hindsight Bias}

Gray (2004) demonstrated that batters' focus of attention varies not only with overall skill level, but also with current performance level. For experts, more attention is allocated to the mechanics of execution during a period of poorer performance (a "slump") and more attention is available to be allocated to the external environment during a period of better performance (a "streak"). The opposite is true for novices. If directing attention to the environment is part of monitoring outcome-relevant information, then greater attention to the environment might support more accurate outcome predictions and less hindsight bias. Therefore, Gray's results suggest that whether such attention-driven maxima in prediction and memory for prediction will occur during better or worse performance should depend on whether one is an expert or novice.

To address this notion, we used a technique described in detail in Gray (2004). Trials 25 through 200 were analyzed for each batter. Total hits numbers of each outcome in the previous 24 trials were calculated for each trial and used as measures of current performance. All trials for which total hits were the same on the previous 24 trials were grouped and the mean difference between the pre and postoutcome marking errors was calculated for each grouping. The same was done for infields, fouls, and misses.

We first analyzed accuracy of preoutcome markings (i.e., initial predictions) as a function of performance. Table 2 shows correlations between mean preoutcome marking errors and the number of each particular outcome in the previous 24 at-bats. For experts there was a negative correlation between $M R E$ and number of hits in the previous 24 at-bats. The correlation grew less negative with poorer outcomes and became positive for misses. Thus experts made their most accurate predictions when performing well. Novices showed the opposite pattern-a positive correlation between $M R E$ and number of hits, growing smaller for infields and fouls, and becoming negative for misses. Thus novices made their most accurate predictions when performing poorly.

Such a pattern might result from the fact, revealed in Table 1, that experts tended to predict more hits whereas novices tended to predict more misses. However, the same pattern emerged in hindsight bias, with experts less susceptible when performing better and novices less susceptible when performing worse. Table 3 shows correlations between the pre-post radial error difference (magnitude of hindsight bias) and the number of each of the four outcomes in the previous 24 trials. Novices showed a positive correlation between hits and magnitude of hindsight bias but a negative correlation between misses and magnitude

Table 3

Correlations Between the Magnitude of Hindsight Bias and Current Performance Level for Each Outcome

\begin{tabular}{llllll}
\hline & \multicolumn{2}{c}{ Novices } & & \multicolumn{2}{c}{ Experts } \\
\cline { 2 - 3 } \cline { 5 - 6 } Outcome & \multicolumn{1}{c}{$M R E$} & \multicolumn{1}{c}{ SME } & & \multicolumn{1}{c}{ MRE } & \multicolumn{1}{c}{$S M E$} \\
\hline Hit & $r(9)=0.67^{*}$ & $r(9)=0.63^{*}$ & & $r(13)=-0.70^{*}$ & $r(13)=-0.58^{*}$ \\
Infield & $r(10)=0.37$ & $r(10)=0.29$ & & $r(11)=-0.32$ & $r(11)=-0.38$ \\
Foul & $r(10)=-0.10$ & $r(10)=0.07$ & & $r(10)=0.20$ & $r(10)=0.31$ \\
Miss & $r(13)=-0.56^{*}$ & $r(13)=-0.60^{*}$ & & $r(7)=0.61^{*}$ & $r(7)=0.65^{*}$ \\
${ }^{*} p<.05$. & & & & &
\end{tabular}




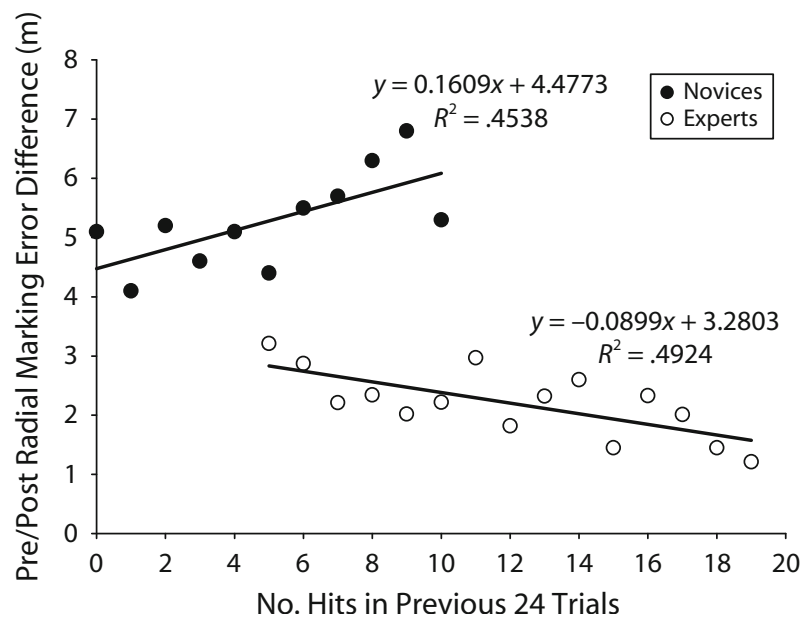

Figure 3. Relationship between current performance level and hindsight bias in experts and novices. The mean difference between $M R E$ for the pre-outcome and postoutcome markings is plotted as a function of the number of hits in the previous 24 trials. The solid line is the best fitting linear curve.

of hindsight bias. In contrast, experts showed a negative correlation between hits and magnitude of hindsight bias but a positive correlation between misses and magnitude of hindsight bias. Figure 3 shows scatterplots of these data. Similar results occurred in analyses of $S M E$ s, suggesting that the pattern is not simply due to differences in the absolute magnitudes of marking errors between experts and novices.

The results indicate that experts' and novices' attention to cues used to make outcome predictions varies as a function of how well they are performing. When experts are performing better, predictions increase in accuracy and hindsight bias decreases, suggesting they are attending more to cues associated with outcome predictions, as might be expected from common-coding theory. When novices are performing better, predictions decrease in accuracy and hindsight bias increases, suggesting they are attending less to such cues. What are novices attending to instead? Successful performance for unskilled individuals requires attention to the step-by-step control of execution (Beilock, Carr, et al., 2002). According to the new index provided by the magnitude of hindsight bias, when novices are performing better, they are attending more to controlling the steps of execution and less to cues about what effects will be generated by those steps, a finding not anticipated by common-coding theory (but quite consistent with previous work on attention).

\section{CONCLUSIONS}

We began with the simple intuition that experts at sensorimotor tasks should be better at predicting the consequences of their own performance than novices. Walt Frazier, the great New York Knicks guard, was famous for shouting "Fall back, baby" just as he released a long jump shot he knew would be good. Major league batters react with disappointment or elation well before the pop-up is caught or the long ball clears the fence. But when we sought empirical proof for this intuition, we did not find it. Nor did we find a theoretical account of how predictions are made.

Together, common-coding and attentional theories provide a framework for generating such an account, at least for experts. As practice accumulates, links between expected feedback about outcome and the processes that produce the outcome should strengthen (Prinz, 1997; Repp \& Knoblich, 2004) — experts have more and more direct experience of the consequences of their actions. Furthermore, as skill increases, attention is progressively removed from controlling the steps of execution (Beilock, Carr, et al., 2002; Gray, 2004), affording a shift to generating and monitoring outcome-relevant information. This shift of attention could provide the basis for establishing the processes that common-coding theory holds to underlie control of sensorimotor performance - a focus of attention on generating expected perceptual feedback and then monitoring the environment to confirm that the expected changes have occurred.

Nested within this long-term trend is shorter-term variation. Experts' greater versus lesser success is coupled with a tighter versus weaker linkage between outcome information and the motor codes generating the action, as well as greater attentional resources released from execution to devote to monitoring outcome-relevant information in advance of the outcome itself. When experts are performing poorly, however, they appear to revert to the linkage and/or attention deployment of an earlier level of skill in which the focus is relatively more upon step-by-step execution and less upon outcome-relevant information. Thus common-coding and attentional theories together provide a unified explanation for the performance of experts and their outcome predictions when they are performing well-both result from a focus of attention on generating outcomes and monitoring for perceptual evidence that the outcome has occurred. This leaves for future research the question of exactly how outcome predictions are made by novices - and by experts in the doldrums of a slump.

\section{AUTHOR NOTE}

This work was supported by the National Science Foundation Faculty Early Career Development Program Award No. 0239657 to R.G. and IES Grant R305H050004 and NSF Grant BCS-0601148 to S.L.B. Correspondence concerning this article should be addressed to R. Gray, Department of Applied Psychology, Arizona State University, 7001 E. Williams Field Road, Building 140, Mesa, AZ 85212 (e-mail: robgray@ asu.edu).

Note-Accepted by DavidA. Balota's editorial team.

\section{REFERENCES}

Bahill, A. T., \& Karnavas, W. J. (1993). The perceptual illusion of baseball's rising fastball and breaking curveball. Journal of Experimental Psychology: Human Perception \& Performance, 19, 3-14.

Beilock, S. L., Bertenthal, B. I., McCoy, A. M., \& Carr, T. H. (2004). Haste does not always make waste: Expertise, direction of attention, and speed versus accuracy in performing sensorimotor skills. Psychonomic Bulletin \& Review, 11, 373-379. 
BeIlock, S. L. \& CARR, T. H. (2001). On the fragility of skilled performance: What governs choking under pressure? Journal of Experimental Psychology: General, 130, 701-725.

Beilock, S. L., Carr, T. H., MacMahon, C., \& Starkes, J. L. (2002). When paying attention becomes counterproductive: Impact of divided versus skill-focused attention on novice and experienced performance of sensorimotor skills. Journal of Experimental Psychology: Applied, 8, 6-16.

BeIlock, S. L., Wierenga, S. A., \& CARr, T. H. (2002). Expertise, attention and memory in sensorimotor skill execution: Impact of novel task constraints on dual-task performance and episodic memory. Quarterly Journal of Experimental Psychology, 55A, 1211-1240.

Craik, F. I. M., Govoni, R., NAVEh-BENJAMIN, M., \& ANDERSON, N. D. (1996). The effects of divided attention on encoding and retrieval processes in human memory. Journal of Experimental Psychology: General, 125, 159-180.

FISCHHOFF, B. (1975). Hindsight not equal to foresight: Effect of outcome knowledge on judgment under uncertainty. Journal of Experimental Psychology: Human Perception \& Performance, 1, 288-299.

GRAY, R. (2002a). Behavior of college baseball players in a virtual batting task. Journal of Experimental Psychology: Human Perception \& Performance, 28, 1131-1148.

GRAY, R. (2002b). "Markov at the Bat": A model of cognitive processing in baseball batters. Psychological Science, 13, 543-548.

GRAY, R. (2004). Attending to the execution of a complex sensorimotor skill: Expertise differences, choking and slumps. Journal of Experimental Psychology: Applied, 10, 42-54.
JACKSON, R. C., AshFord, K. J., \& Norsworthy, G. (2006). Attentional focus, dispositional reinvestment and skilled motor performance under pressure. Journal of Sport \& Exercise Psychology, 28, 48-68.

KNOBLICH, G., \& Flach, R. (2001). Predicting the effects of actions: Interactions of perception and action. Psychological Science, 12, 467-472.

Masters, R. S. W. (1992). Knowledge, knerves and know-how: The role of explicit versus implicit knowledge in the breakdown of a complex motor skill under pressure. British Journal of Psychology, 83, 343-358.

Perkins-Ceccato, N., Passmore, S. R., \& Lee, T. D. (2003). Effects of focus of attention depend on golfers' skill. Journal of Sports Sciences, 21, 593-600.

PoWERS, W. T. (1973). Feedback: Beyond behaviorism. Science, 179, 351-356.

PrINZ, W. (1997). Perception and action planning. European Journal of Cognitive Psychology, 9, 129-154.

REPP, B. H., \& KNOBLICH, G. (2004). Perceiving action identity: How pianists recognize their own performances. Psychological Science, 15, 605-609.

Tresilian, J. R., \& Lonergan, A. (2002). Intercepting a moving target: Effects of temporal precision constraints and movement amplitude. Experimental Brain Research, 142, 193-207.

WULF, G., \& PRINZ, W. (2001). Directing attention to movement effects enhances learning: A review. Psychonomic Bulletin \& Review, 8, 648-660.

(Manuscript received December 28, 2005; revision accepted for publication August 24, 2006.) 